\title{
Effectiveness of Interventions to Improve the Anticholinergic Prescribing Practice in Older Adults: A Systematic Review
}

\author{
Mohammed S. Salahudeen ${ }^{1, * \mathbb{D}}$, Adel Alfahmi 1,2 ${ }^{\mathbb{D}}$, Anam Farooq ${ }^{3}$, Mehnaz Akhtar ${ }^{4}$, Sana Ajaz $^{4}$, \\ Saud Alotaibi ${ }^{5}$, Manal Faiz ${ }^{6}$ and Sheraz Ali ${ }^{1}$ (D)
}

check for

updates

Citation: Salahudeen, M.S.; Alfahmi,

A.; Farooq, A.; Akhtar, M.; Ajaz, S.;

Alotaibi, S.; Faiz, M.; Ali, S.

Effectiveness of Interventions to

Improve the Anticholinergic

Prescribing Practice in Older Adults:

A Systematic Review. J. Clin. Med.

2022, 11, 714. https://doi.org/

$10.3390 /$ jcm 11030714

Academic Editor: Francesco

Mattace-Raso

Received: 31 December 2021

Accepted: 25 January 2022

Published: 28 January 2022

Publisher's Note: MDPI stays neutral with regard to jurisdictional claims in published maps and institutional affiliations.

Copyright: (C) 2022 by the authors. Licensee MDPI, Basel, Switzerland This article is an open access article distributed under the terms and conditions of the Creative Commons Attribution (CC BY) license (https:// creativecommons.org/licenses/by/ $4.0 /)$.
1 School of Pharmacy and Pharmacology, University of Tasmania, Hobart 7001, Australia; adel.alfahmi@utas.edu.au (A.A.); sheraz.ali@utas.edu.au (S.A.)

2 Department of Pharmacy, East Jeddah General Hospital, Ministry of Health, Jeddah 22253, Saudi Arabia

3 Pharmaceutical Care Department, Dr Sulaiman Al-Habib Hospital, Riyadh 12214, Saudi Arabia; anamfarooq91@gmail.com

4 Shifa College of Pharmaceutical Sciences, Shifa Tmaeer-e-Millat University, Islamabad 44000, Pakistan; mehnaz.akhtar70@gmail.com (M.A.); sanaajaz.edu@gmail.com (S.A.)

5 Pharmaceutical Care Services, King Saud Medical City, Ministry of Health, Riyadh 12746, Saudi Arabia; saud_2007_sa@hotmail.com

6 Azra Naheed Medical College, Superior University, Lahore 55150, Pakistan; manalfaiz47@gmail.com

* Correspondence: mohammed.salahudeen@utas.edu.au

\begin{abstract}
Background: Pharmacotherapy in older adults is one of the most challenging aspects of patient care. Older people are prone to drug-related problems such as adverse effects, ineffectiveness, underdosage, overdosage, and drug interactions. Anticholinergic medications are associated with poor outcomes in older patients, and there is no specific intervention strategy for reducing drug burden from anticholinergic activity medications. Little is known about the effectiveness of current interventions that may likely improve the anticholinergic prescribing practice in older adults. Aims: This review seeks to document all types of interventions aiming to reduce anticholinergic prescribing among older adults and assess the current evidence and quality of existing single and combined interventions. Methods: We systematically searched MEDLINE, Embase, Cochrane Central Register of Controlled Trials, CINAHL, and PsycINFO from January 1990 to August 2021. Only studies that examined the effect of interventions in older people focused on improving compliance with anticholinergic prescribing guidelines with quantifiable data were included. The primary outcome of interest was to find the effectiveness of interventions that enhance the anticholinergic prescribing practice in older adults. Results: We screened 3168 records and ended up in 23 studies that met the inclusion criteria. We found only single-component interventions to reduce anticholinergic prescribing errors in older people. Pharmacists implemented interventions without collaboration in nearly half of the studies $(n=11)$. Medication review $(43 \%)$ and education provision $(26 \%)$ to healthcare practitioners were the most common interventions. Sixteen studies $(70 \%)$ reported significant reductions in anticholinergic prescribing errors, whereas seven studies ( $30 \%$ ) showed no significant effect. Conclusion: This systematic review suggests that healthcare practitioner-oriented interventions have the potential to reduce the occurrence of anticholinergic prescribing errors in older people. Interventions were primarily effective in reducing the burden of anticholinergic medications and assisting with deprescribing anticholinergic medications in older adults.
\end{abstract}

Keywords: anticholinergics; intervention; prescribing; older people

\section{Introduction}

Prescribing medications among older adults is recognised as a challenging task and an essential practice that needs to be continuously monitored, assessed, and refined accordingly. Moreover, it is based on understanding clinical pharmacology principles, knowledge 
about medicines, and particularly the experience and empirical knowledge of the prescribers [1,2]. Clinicians face several challenges while prescribing medications among older adults, and the prescribing of potentially inappropriate medications (PIMs) for this age group is prevalent [3]. The available epidemiological data show that up to $20 \%$ of older patients in outpatient settings and $59 \%$ of hospitalised older patients consume at least one PIM [4-8]. Adverse effects in older people due to inappropriate prescribing are prevalent, leading to increased hospital admissions and mortality [9].

Medications that possess anticholinergic activity are a class of PIMs widely prescribed for various clinical conditions in older adults [10,11]. Older people are particularly vulnerable to the adverse effects from medicines with anticholinergic-type effects [12,13]. Most medications commonly prescribed to older people are not routinely recognised as having anticholinergic activity, and empirically, clinicians prescribe these medicines based on their anticipated therapeutic benefits while overlooking the risk of cumulative anticholinergic burden [14-16]. Anticholinergic burden refers to the cumulative effect of taking multiple medications with anticholinergic activity $[17,18]$. There is no gold standard approach available to quantify and determine whether an acceptable range of anticholinergic drug burden exists in older adults $[19,20]$. The central adverse effects of anticholinergic medications are attributed to the excess blocking of cholinergic receptors within the central nervous system (CNS) [16]. The commonly reported central adverse effects are cognitive impairment, headache, reduced cognitive function, anxiety, and behavioural disturbances [16]. The common peripheral adverse effects of anticholinergic medications are hyperthermia, reduced saliva and tear production, urinary retention, constipation, and tachycardia [16].

Anticholinergic medications are associated with poor outcomes in older patients, but there is no specific intervention strategy for reducing anticholinergic drug exposure [21]. There is little evidence that medication review could be a promising strategy in reducing the drug burden in older people [22,23]. Medical practitioner-led and pharmacist-led medication reviews have earlier been reported as a standard practice for reducing anticholinergic drug exposure [24,25]. Pharmacist-led medication review has recently been found to be ineffective among older patients of the Northern Netherlands [25]. A few metaanalyses have also reported the lack of effectiveness of different types of medication reviews on mortality and hospitalisation outcomes [26-28]. Multidisciplinary strategies such as patient-centred, pharmacist-physician intervention are also recognised as promising for improving medication use in older patients at risk [29]. Another intervention strategy, i.e., the SÄKLÄK project, had some effects on the PIMs prescription and reduced potential medication-related problems [30]. The SÄKLÄK project is a multi-professional intervention model to improve medication use in older people [30], and it consists of self-assessment using a questionnaire, peer-reviewed by experienced healthcare professionals, feedback report provided by experienced healthcare professionals, and an improvement plan [30].

Interventions to improve prescribing practice more generally have been the subject of many studies and are frequently targeted according to the type of error [31,32]. It is crucial to explore which interventions have effectively changed prescribing practices and optimised patient outcomes while minimising healthcare costs. However, little is known about the effectiveness of existing interventions at improving the anticholinergic prescribing practice for older adults. Hence, this review seeks to document all types of interventions aiming to reduce anticholinergic prescribing errors among older adults and assess the evidence of existing single and combined interventions.

\section{Methods}

The Preferred Reporting Items for Systematic Reviews and Meta-Analyses (PRISMA) guideline was applied to report the findings of this systematic review [33].

\subsection{Data Sources and Search Strategy}

The following databases were examined between January 1990 and August 2021: Ovid MEDLINE, Ovid EMBASE, Ovid PsycINFO, and the Cochrane Central Register of 
Controlled Trials (CENTRAL). A comprehensive electronic search was performed using appropriate keywords on anticholinergics, older people, and interventions to retrieve the relevant studies. The search was limited to the English language and humans. A detailed MEDLINE search strategy is presented in Supplementary Table S1. Citation analysis was performed in Google Scholar and Web of Science to track the prospective citing of references of the selected articles.

\subsection{Study Screening and Selection}

The title, abstract, and full text of each potentially relevant article were independently screened by two authors (M.S. and S.A.) for eligibility of inclusion in this review. Any discrepancies were resolved by a third author (A.A.), and decisions were made by consensus.

\subsection{Inclusion Criteria}

The primary outcome of interest was single and multicomponent interventions that improve anticholinergic prescribing practice or reduce adverse drug events due to the consumption of anticholinergic medications. Single-component intervention consists of only one intervention activity, such as medication review [34]. Multicomponent intervention refers to the combination of various components in a single intervention [35], such as medication review and the provision of education [34]. All interventions (e.g., medication review, educational detailing visits for physicians, nurses, and aides, pocket-sized educational cards along with clinical vignettes, educational internet site, and detailing session with physicians) performed by any healthcare professional targeting participants of either sex, mean age $\geq 65$ years, and admitted to any healthcare setting were included. We included pre/post or experimental studies that employed a control group.

\subsection{Exclusion Criteria}

We excluded the following studies: review articles, case reports, and case series. We also excluded studies that were conducted in languages other than English.

\subsection{Data Extraction and Synthesis}

Two reviewers (M.S. and S.A.) independently reviewed and extracted the data from the eligible studies according to a standardised format based on variables of interest, such as the study population, study design and duration, mean age, major findings, and intervention characteristics (type of intervention and implementation). The study selection process is illustrated in Figure 1.

\subsection{Quality Assessment}

The quality of the included studies was critically appraised. The Cochrane Risk of Bias tool [36] was used to assess the methodological quality of the randomised controlled trials (RCTs). The Newcastle-Ottawa scale was used to assess the quality of the non-RCTs [37], which is based on three domains: the selection of study groups, comparability of cohorts and assessment of outcome (cohort studies), or comparability of case and controls and ascertainment of exposure (case-control studies). The thresholds for categorising and interpreting the Newcastle-Ottawa scale domains were described in Supplementary Table S2. The Cochrane Risk of Bias tool results for included RCTs were described in Supplementary Table S3. Studies were not excluded based on the risk of bias or quality assessment. 
IDENTIFICATION

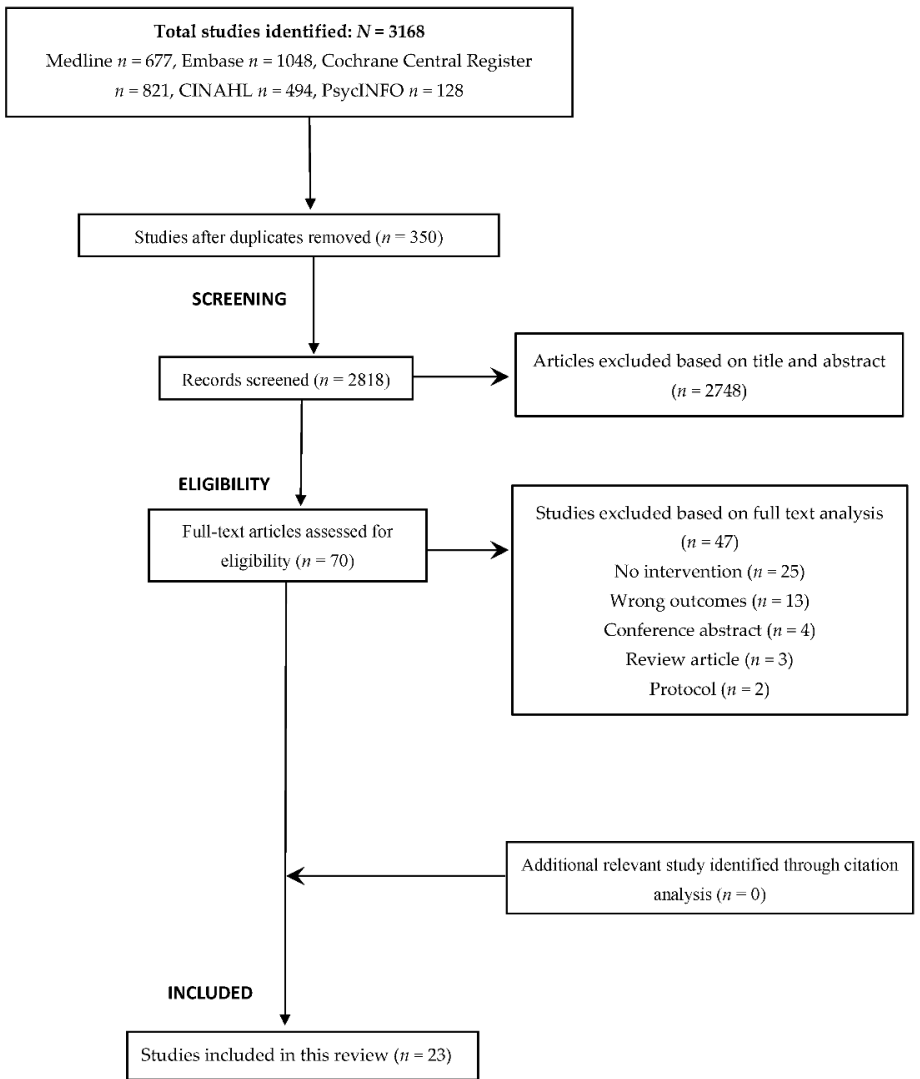

Figure 1. PRISMA flow diagram of the study selection process and citation analysis.

\section{Results}

The primary electronic search identified a total of 3168 studies from the five databases. Using EndNote X9 (Thomson Reuters), we eliminated 350 duplicate studies, and the remaining 2818 studies were examined to determine their relevance for inclusion. Of those, only 70 were found to be eligible for full-text analysis. Subsequently, 47 studies were excluded as they failed to meet the predefined inclusion criteria. No potential studies were identified from the citation analysis. Finally, a total of 23 studies that investigated the effectiveness of anticholinergic prescribing practice in older adults were included in this review (Figure 1).

\subsection{Overview of the Included Studies}

Table 1 provides the qualitative summary of the included studies, mainly showcasing the type of interventions, and Table 2 illustrates an overview of the quantitative summary of the studies based on study design, setting, sample size, study duration and follow-up, outcome measure (control/pre and intervention/post), significant association (+ or - ), and statistical tests.

The countries of origin were USA $(n=5)$ [29,38-41], Australia $(n=4)[22,23,42,43]$, Finland $(n=2)[44,45]$, Norway $(n=2)$ [21,46], Ireland [47], New Zealand [48], Belgium [49], Spain [50], Sweden [51], Sweden [30], France [52], Italy [53], Taiwan [54], and The Netherlands [55].

The study settings included hospitals $(n=7)[40,41,44,46,50,52,53]$ community/primary care $(n=7)[22,30,38,47,49,51,55]$ and nursing homes/aged care facilities $(n=9)[21,23,29,39,42,43,45,48,54]$. There were ten cross-sectional studies $[22,38,40,42-44,46,51,52,54]$, six nonrandomised or pre/post studies [30,39,47,48,50,53], and seven RCTs [21,23,29,41,45,49,55]. The studies included in this study had sample sizes ranging from 46 to 46,078 study subjects. The average age of the participants varied between 65 and 87.5 years, and the proportion of the female subjects was $39.0-77 \%$. 
Table 1. The qualitative summary of included studies.

\begin{tabular}{|c|c|c|c|c|}
\hline $\begin{array}{c}\text { Author, Year, } \\
\text { Country }\end{array}$ & Study Design & Intervention & Description of Intervention(s) & Effect on Outcome/Key Findings \\
\hline $\begin{array}{l}\text { Riordan et al., } \\
\text { 2019, Ireland [47] }\end{array}$ & $\begin{array}{l}\text { Convergent } \\
\text { parallel } \\
\text { mixed-methods } \\
\text { design (before } \\
\text { and after) }\end{array}$ & $\begin{array}{c}\text { Academic } \\
\text { Detailing } \\
\text { (pharmacist-led) }\end{array}$ & $\begin{array}{l}\text { Pharmacist conducted face-to-face education } \\
\text { sessions and small focus group academic detailing sessions of } \\
19-48 \text { min with physicians. }\end{array}$ & $\begin{array}{c}\text { Pharmacist-led academic detailing intervention was acceptable } \\
\text { to GPs. } \\
\text { Behavioural Change: awareness of non-pharmacological } \\
\text { methods in treating urinary incontinence. } \\
\text { Knowledge Gain: intervention served to refresh their } \\
\text { knowledge }\end{array}$ \\
\hline $\begin{array}{l}\text { Ailabouni et al., } \\
\text { 2019, New } \\
\text { Zealand [48] }\end{array}$ & $\begin{array}{l}\text { A single group } \\
\text { (pre-and } \\
\text { post-comparison) } \\
\text { feasibility study }\end{array}$ & $\begin{array}{l}\text { Medication } \\
\text { review } \\
\text { (deprescribing) }\end{array}$ & $\begin{array}{c}\text { A collaborative pharmacist-led medication review with GPs was } \\
\text { employed. } \\
\text { New Zealand registered pharmacists used peer-reviewed } \\
\text { deprescribing guidelines. The cumulative use of anticholinergic } \\
\text { and sedative medicines for each participant was quantified } \\
\text { using the DBI. }\end{array}$ & $\begin{array}{c}\text { Deprescribing resulted in a significant reduction in falls, } \\
\text { depression and frailty scores, and adverse drug reactions. No } \\
\text { improvement in cognition and quality of life. } \\
\text { Total regular medicines use reduced statistically, by a mean } \\
\text { difference of } 2.13 \text { medicines per patient, among patients where } \\
\text { deprescribing was initiated. }\end{array}$ \\
\hline $\begin{array}{l}\text { Toivo et al., 2019, } \\
\text { Belgium [49] }\end{array}$ & Cluster RCT & $\begin{array}{l}\text { Care coordination } \\
\text { intervention } \\
\text { (coordinated } \\
\text { medication risk } \\
\text { management) }\end{array}$ & $\begin{array}{l}\text { Practical nurses were trained to make the preliminary } \\
\text { medication risk assessment during home visits and report } \\
\text { findings to the coordinating pharmacist. The coordinating } \\
\text { pharmacist prepared the cases for the triage meeting with the } \\
\text { physician and home care nurse to decide further actions. }\end{array}$ & $\begin{array}{l}\text { No significant impact on the medication risks between the } \\
\text { intervention and the control group. } \\
\text { The per-protocol analysis indicated a tendency for effectiveness, } \\
\text { particularly in optimising central nervous system medication } \\
\text { use. }\end{array}$ \\
\hline $\begin{array}{l}\text { Hernandez et al., } \\
\text { 2020, Spain [50] }\end{array}$ & $\begin{array}{l}\text { Prospective } \\
\text { pre-and post- } \\
\text { interventional } \\
\quad \text { study }\end{array}$ & $\begin{array}{l}\text { Medication } \\
\text { review }\end{array}$ & $\begin{array}{c}\text { Pharmacists reviewed the medications and detected } \\
\text { drug-related problems using the Drug Burden Index (DBI) tool. } \\
\text { Their recommendations were communicated to the physician } \\
\text { via telephone, weekly meetings, and email. Further review was } \\
\text { conducted at the weekly meeting between physician and } \\
\text { pharmacist. }\end{array}$ & $\begin{array}{l}\text { Statistically significant differences were found between pre- and } \\
\text { post-intervention in NPI at admission, drug-related problems, } \\
\text { MAI criteria (interactions, dosage and duplication), and mean } \\
\text { (SD) DBI score. }\end{array}$ \\
\hline $\begin{array}{l}\text { Lenander et al., } \\
\text { 2018, Sweden [51] }\end{array}$ & Cross-sectional & $\begin{array}{l}\text { Medication } \\
\text { Review }\end{array}$ & $\begin{array}{l}\text { Clinical Pharmacist led medication review to assess the } \\
\text { prevalence of DRPs and recommendations to discontinue, } \\
\text { followed by team-based discussions with general practitioners } \\
\text { (GPs) and nurses }\end{array}$ & $\begin{array}{l}\text { It shows that the medication reviews decreased the use of } \\
\text { potentially inappropriate medication. }\end{array}$ \\
\hline $\begin{array}{l}\text { Weichert et al., } \\
\text { 2018, Finland [44] }\end{array}$ & $\begin{array}{l}\text { Multicentre } \\
\text { observational } \\
\text { study }\end{array}$ & $\begin{array}{l}\text { Medication } \\
\text { Review }\end{array}$ & $\begin{array}{c}\text { Medication review was conducted for ACB in patients at the } \\
\text { time of admission and discharge }\end{array}$ & $\begin{array}{l}21.1 \% \text { of patients had their ACB reduced. There is considerable } \\
\text { scope for improvement of prescribing practices in older people. }\end{array}$ \\
\hline
\end{tabular}


Table 1. Cont.

\begin{tabular}{|c|c|c|c|c|}
\hline $\begin{array}{l}\text { Author, Year, } \\
\text { Country }\end{array}$ & Study Design & Intervention & Description of Intervention(s) & Effect on Outcome/Key Findings \\
\hline $\begin{array}{l}\text { Lenander et al., } \\
\text { 2017, Sweden [30] }\end{array}$ & $\begin{array}{l}\text { Interventional } \\
\text { pilot study }\end{array}$ & $\begin{array}{l}\text { SÄKLÄK project, } \\
\text { a developed } \\
\text { intervention } \\
\text { model }\end{array}$ & $\begin{array}{l}\text { Multi-professional intervention model created to improve } \\
\text { medication safety for elderly }\end{array}$ & $\begin{array}{l}\text { Significant decrease in the prescription of anticholinergic drugs } \\
\text { indicated the SÄKLÄK intervention is effective in reducing } \\
\text { potential DRPs }\end{array}$ \\
\hline $\begin{array}{l}\text { Moga et al., 2017, } \\
\text { USA [29] }\end{array}$ & $\begin{array}{l}\text { Parallel arm } \\
\text { Randomised } \\
\text { Interventional } \\
\quad \text { study }\end{array}$ & $\begin{array}{c}\text { Targeted } \\
\text { medication } \\
\text { therapy } \\
\text { management } \\
\text { intervention }\end{array}$ & $\begin{array}{l}\text { Targeted patient-centred pharmacist-physician team medication } \\
\text { therapy management intervention was used to reduce the use of } \\
\text { inappropriate anticholinergic medications in older patients. }\end{array}$ & $\begin{array}{l}\text { The targeted medication therapy management intervention } \\
\text { resulted in improvement in anticholinergic medication } \\
\text { appropriateness and reduced the use of inappropriate } \\
\text { anticholinergic medications in older patients. }\end{array}$ \\
\hline $\begin{array}{l}\text { Lagrange et al., } \\
\text { 2017, France [52] }\end{array}$ & $\begin{array}{l}\text { Retrospective } \\
\text { study }\end{array}$ & $\begin{array}{l}\text { A context-aware } \\
\text { pharmaceutical } \\
\text { analysis tool }\end{array}$ & $\begin{array}{l}\text { A context-aware computerised decision-support system } \\
\text { designed to automatically compare prescriptions recorded in } \\
\text { computerised patient files against the main consensual } \\
\text { guidelines for medical management in older adults. }\end{array}$ & $\begin{array}{l}\text { Prescription of anticholinergics was significantly decreased } \\
\qquad(28 \%) .\end{array}$ \\
\hline $\begin{array}{l}\text { Carnahan et al., } \\
\text { 2017, USA [39] }\end{array}$ & $\begin{array}{l}\text { Quasi- } \\
\text { experimental } \\
\text { study design }\end{array}$ & $\begin{array}{l}\text { Educational } \\
\text { program on } \\
\text { medication use }\end{array}$ & $\begin{array}{l}\text { IA-ADAPT/CMS Partnership is an evidence-based training } \\
\text { program to improve dispensing drugs for elderly }\end{array}$ & $\begin{array}{l}\text { Suggests that the IA-ADAPT and the CMS Partnership } \\
\text { improved medication use with no adverse impact on BPSD. }\end{array}$ \\
\hline $\begin{array}{l}\text { McLarin et al., } \\
\text { 2016, Australia } \\
\quad[43]\end{array}$ & $\begin{array}{l}\text { Retrospective } \\
\text { study }\end{array}$ & RMMR & $\begin{array}{c}\text { Impact of RMMRs on anticholinergic burden quantified by } \\
\text { seven anticholinergic risk scales }\end{array}$ & $\begin{array}{l}\text { Demonstrated that RMMRs are effective in reducing ACM } \\
\text { prescribing in elderly }\end{array}$ \\
\hline
\end{tabular}


Table 1. Cont.

\begin{tabular}{|c|c|c|c|c|}
\hline $\begin{array}{l}\text { Author, Year, } \\
\text { Country }\end{array}$ & Study Design & Intervention & Description of Intervention(s) & Effect on Outcome/Key Findings \\
\hline $\begin{array}{l}\text { Kersten et al., } \\
\text { 2015, Norway } \\
{[46]}\end{array}$ & $\begin{array}{l}\text { Retrospective } \\
\text { study }\end{array}$ & $\begin{array}{l}\text { Medication } \\
\text { review }\end{array}$ & $\begin{array}{l}\text { Investigated the clinical impact of PIMs in acutely hospitalised } \\
\text { older adults. }\end{array}$ & Anticholinergic prescriptions were reduced from $39.2 \%$ to $37.9 \%$ \\
\hline $\begin{array}{l}\text { Juola et al., 2015, } \\
\text { Finland [45] }\end{array}$ & Cluster RCT & $\begin{array}{l}\text { Educational } \\
\text { intervention }\end{array}$ & $\begin{array}{l}\text { Nursing staff working in the intervention wards received two } \\
\text { 4-h interactive training sessions based on constructive learning } \\
\text { theory to recognise harmful medications and adverse drug } \\
\text { events. }\end{array}$ & $\begin{array}{l}\text { No significant differences in the change in prevalence of } \\
\text { anticholinergic drugs. }\end{array}$ \\
\hline $\begin{array}{l}\text { Kersten et al., } \\
\text { 2013, Norway } \\
\text { [21] }\end{array}$ & $\mathrm{RCT}$ & $\begin{array}{l}\text { Multidisciplinary } \\
\text { drug review }\end{array}$ & $\begin{array}{l}\text { Single Blind MDRD was conducted that recruited long-term } \\
\text { nursing home residents with a total ADS score of greater than or } \\
\text { equal to } 3\end{array}$ & $\begin{array}{l}\text { After } 8 \text { weeks, the median ADS score was significantly reduced } \\
\text { from } 4 \text { to } 2 \text { in the intervention group. The largest improvement } \\
\text { in immediate recall after } 8 \text { weeks was observed in the five } \\
\text { patients in the intervention group who had their ADS score } \\
\text { reduced to } 0\end{array}$ \\
\hline $\begin{array}{l}\text { Ghibelli et al., } \\
\text { 2013, Italy [53] }\end{array}$ & $\begin{array}{l}\text { Pre, } \\
\text { post-intervention } \\
\text { study }\end{array}$ & $\begin{array}{l}\text { INTERcheck } \\
\text { CPSS }\end{array}$ & $\begin{array}{l}\text { INTERcheck is a CPSS developed to optimise drug prescription } \\
\text { for older people with multimorbidity and minimise the } \\
\text { occurrence of adverse drug reactions. }\end{array}$ & $\begin{array}{l}\text { The use of INTERCheck was associated with a significant } \\
\text { reduction in PIMs and new-onset potentially severe DDIs. }\end{array}$ \\
\hline $\begin{array}{l}\text { Yeh et al., 2013, } \\
\text { Taiwan [54] }\end{array}$ & $\begin{array}{l}\text { Prospective } \\
\text { case-control } \\
\text { study }\end{array}$ & $\begin{array}{l}\text { Educational } \\
\text { program for } \\
\text { primary care } \\
\text { physicians }\end{array}$ & $\begin{array}{c}\text { Educational program for primary care physicians serving in } \\
\text { Veterans' Homes, focusing on anticholinergic adverse reactions } \\
\text { in geriatrics and the CR-ACHS }\end{array}$ & $\begin{array}{l}\text { CR-ACHS was significantly reduced in the intervention group } \\
\text { at 12-week follow-up. }\end{array}$ \\
\hline $\begin{array}{l}\text { Boustani et al., } \\
\text { 2012, USA [41] }\end{array}$ & $\mathrm{RCT}$ & $\begin{array}{l}\text { CDSS Alert } \\
\text { (anticholinergic } \\
\text { discontinuation) }\end{array}$ & $\begin{array}{c}\text { CDSS alert system sends an interruptive alert if any of the } 18 \\
\text { anticholinergics were prescribed, recommending stopping the } \\
\text { drug, suggesting an alternative, or recommending dose } \\
\text { modification. }\end{array}$ & $\begin{array}{l}\text { Physicians receiving the CDSS issued more discontinuation } \\
\text { orders of definite anticholinergics, but the results were not } \\
\text { statistically significant. Results suggest that human interaction } \\
\text { may play an important role in accepting recommendations } \\
\text { aimed at improving the care of hospitalised older adults with CI. }\end{array}$ \\
\hline
\end{tabular}


Table 1. Cont.

\begin{tabular}{|c|c|c|c|c|}
\hline $\begin{array}{l}\text { Author, Year, } \\
\text { Country }\end{array}$ & Study Design & Intervention & Description of Intervention(s) & Effect on Outcome/Key Findings \\
\hline $\begin{array}{l}\text { Gnjidic et al., } \\
\text { 2010, Australia } \\
\text { [23] }\end{array}$ & Cluster RCT & $\begin{array}{l}\text { Medication } \\
\text { review }\end{array}$ & $\begin{array}{l}\text { The study intervention included a letter and phone call to GPs, } \\
\text { using DBI to prompt them to consider dose reduction or } \\
\text { cessation of anticholinergic and sedative medications. }\end{array}$ & $\begin{array}{l}\text { At follow-up, a DBI change was observed in } 16 \text { participants. } \\
\text { DBI decreased in } 12 \text { participants, } 6(19 \%) \text { in the control group, } \\
\text { and } 6(32 \%) \text { in the intervention group. }\end{array}$ \\
\hline $\begin{array}{l}\text { Castelino et al., } \\
\text { 2010, Australia } \\
\text { [22] }\end{array}$ & $\begin{array}{l}\text { Retrospective } \\
\text { study }\end{array}$ & $\begin{array}{l}\text { Medication } \\
\text { reviews by } \\
\text { pharmacist }\end{array}$ & $\begin{array}{l}\text { HMR by pharmacists for leads to an improvement in the use of } \\
\text { medications }\end{array}$ & $\begin{array}{l}\text { DBI and PIMs identified in } 60.5 \% \text { and } 39.8 \% \text { of the patients. } \\
\text { Significant reduction in the cumulative DBI scores for all } \\
\text { patients was observed following pharmacists' recommendations }\end{array}$ \\
\hline $\begin{array}{l}\text { Starner et al., } \\
\text { 2009, USA [38] }\end{array}$ & $\begin{array}{l}\text { Retrospective } \\
\text { study }\end{array}$ & $\begin{array}{l}\text { Educational } \\
\text { Intervention }\end{array}$ & $\begin{array}{l}\text { Intervention letters were mailed to the physicians for patients } \\
\text { having } \geq 1 \text { DAE claim }\end{array}$ & $\begin{array}{l}\text { Noticeable decrease was observed after a 6-month follow-up of } \\
\text { the intervention in the reduction of DAE claims }(48.8 \%) \\
\text { specifically reduction of anticholinergics }(66.7 \%) \text { was highest }\end{array}$ \\
\hline $\begin{array}{l}\text { van Eijk et al., } \\
\text { 2001, } \\
\text { Netherlands [55] }\end{array}$ & $\mathrm{RCT}$ & $\begin{array}{l}\text { Educational visits } \\
\text { as an individual } \\
\text { and a group for } \\
\text { general } \\
\text { practitioners and } \\
\text { pharmacists }\end{array}$ & $\begin{array}{l}\text { Educational visits used academic detailing to discuss } \\
\text { prescribing of highly anticholinergic antidepressants in elderly } \\
\text { people. }\end{array}$ & $\begin{array}{l}\text { The rate of starting anticholinergic antidepressants in the elderly } \\
\text { reduced } 26 \% \text { (in the individual intervention) and } 45 \% \text { (in the } \\
\text { group intervention) } \\
\text { The use of less anticholinergic antidepressants increased by } 40 \% \\
\text { and } 29 \% \text {, respectively }\end{array}$ \\
\hline
\end{tabular}

MAI, medication appropriateness index; GPs, general practitioners; DBI, drug burden index; NPI, neuropsychiatry inventory; RCT, randomised controlled trial; CDSS, clinical decision support system alert; DAE, drugs to be avoided in the elderly; DRPs, drug-related problems; CI, cognitive impairment; ACB, anticholinergic burden; MDRD, modification of diet in renal disease study equation; ADS, anticholinergic drug scale; PIMs, potentially inappropriate medications; CPSS, computerised prescription support system; DDIs, drug-drug interactions; EHR, electronic health record: DHS, Department of Health Services; ARS, anticholinergic risk scale; CR-ACHS, clinician-rated anticholinergic score; HMR, home medicines review; IA-ADAPT, improving antipsychotic appropriateness in dementia patients; CMS, Centers for Medicare and Medicaid Services Partnership to Improve Dementia Care; BPSD, Behavioural and psychological symptoms of dementia; RMMRs, Residential Medication Management Reviews; ACM, anticholinergic medication. 
Table 2. The quantitative summary of included studies.

\begin{tabular}{|c|c|c|c|c|c|c|c|c|c|c|c|c|}
\hline \multirow{2}{*}{$\begin{array}{l}\text { Author, Year, } \\
\text { Country }\end{array}$} & \multirow{2}{*}{ Study Design } & \multirow{2}{*}{ Setting } & \multirow{2}{*}{ Sample Size } & \multirow{2}{*}{$\begin{array}{c}\text { Mean Age } \\
\text { (Years) }\end{array}$} & \multirow{2}{*}{$\begin{array}{c}\text { Gender } \\
\text { (Female \%) }\end{array}$} & \multirow{2}{*}{$\begin{array}{c}\text { Study } \\
\text { Duration }\end{array}$} & \multirow{2}{*}{ Follow-Up } & \multirow{2}{*}{$\begin{array}{c}\text { Relevant } \\
\text { Outcome(s) }\end{array}$} & \multicolumn{2}{|c|}{ Outcome Measure } & \multirow{2}{*}{$\begin{array}{c}\text { Significant } \\
\text { Association } \\
( \pm)\end{array}$} & \multirow{2}{*}{ Statistical Tests } \\
\hline & & & & & & & & & Control/Pre & Intervention/Post & & \\
\hline $\begin{array}{l}\text { Riordan et al., } \\
\text { 2019, Ireland } \\
\text { [47] }\end{array}$ & $\begin{array}{l}\text { Convergent } \\
\text { parallel } \\
\text { mixed- } \\
\text { methods } \\
\text { design (before } \\
\text { and after) }\end{array}$ & $\begin{array}{l}\text { General } \\
\text { Practice }\end{array}$ & 154 & 75.0 & 72.1 & 5 months & 6 months & $\begin{array}{l}\text { Effects on DBI } \\
\text { and ACB } \\
\text { scores }\end{array}$ & $\begin{array}{c}\text { Patients } \\
\text { having an } \\
\text { ACB score of } \\
0(34 \%)\end{array}$ & $\begin{array}{c}\text { Patients having an } \\
\text { ACB score of } 0 \\
(31 \%) \\
65 \% \text { of patients } \\
\text { did not show any } \\
\text { change } \\
\text { in DBI over time }\end{array}$ & - & $\begin{array}{l}\text { SD, Range, IQR, } \\
\text { Frequency, } \\
\text { Percentages }\end{array}$ \\
\hline $\begin{array}{c}\text { Ailabouni } \\
\text { et al., 2019, } \\
\text { New Zealand } \\
\text { [48] }\end{array}$ & $\begin{array}{l}\text { A single } \\
\text { group (pre- } \\
\text { and post- } \\
\text { comparison) } \\
\text { feasibility } \\
\text { study }\end{array}$ & $\begin{array}{c}\text { Residential } \\
\text { care facilities }\end{array}$ & 46 & 65.0 & 74.0 & 6 months & 2 weeks & $\begin{array}{l}\text { Reduction in } \\
\text { DBI score }\end{array}$ & $\begin{array}{l}\geq 0.5 \text { (median } \\
\mathrm{DBI} \text { ) }\end{array}$ & 0.34 (median DBI) & + & $\begin{array}{c}\text { Wilcox-signed } \\
\text { Rank test (WSR) } \\
t \text {-test } \\
\text { Fisher's exact test }\end{array}$ \\
\hline $\begin{array}{c}\text { Toivo et al., } \\
\text { 2019, Belgium } \\
\text { [49] }\end{array}$ & Cluster RCT & Primary care & 129 & 82.8 & 69.8 & 1 year & 1 year & $\begin{array}{l}\text { Anticholinergic } \\
\text { use }\end{array}$ & $\begin{array}{l}18.8 \% \text { (Anti- } \\
\text { cholinergic } \\
\text { use at } \\
\text { baseline) } \\
18.8 \% \text { (Anti- } \\
\text { cholinergic } \\
\text { use at 12 } \\
\text { months) }\end{array}$ & $\begin{array}{c}29.6 \% \\
\text { (Anticholinergic } \\
\text { use at baseline) } \\
18.5 \% \\
\text { (Anticholinergic } \\
\text { use at } 12 \text { months) }\end{array}$ & - & $\begin{array}{l}\text { Binary logistic } \\
\text { regression, } \\
\text { two-sided } \\
\text { statistical tests }\end{array}$ \\
\hline $\begin{array}{l}\text { Hernandez } \\
\text { et al., 2020, } \\
\text { Spain [50] }\end{array}$ & $\begin{array}{l}\text { Prospective } \\
\text { pre- and post- } \\
\text { interventional } \\
\text { study }\end{array}$ & $\begin{array}{l}\text { Intermediate } \\
\text { care hospital }\end{array}$ & 55 & 84.6 & 60.0 & 12 months & NA & $\begin{array}{l}\text { Anticholinergic } \\
\text { burden per } \\
\text { Drug Burden } \\
\text { Index (DBI) }\end{array}$ & $\begin{array}{c}1.38 \pm 0.7 \\
\text { (Mean DBI) }\end{array}$ & $\begin{array}{c}1.08 \pm 0.7 \\
\text { (Mean DBI) }\end{array}$ & + & $\begin{array}{l}\text { Kolmogorov- } \\
\text { Smirnov test } \\
\text { Student's } t \text {-test }\end{array}$ \\
\hline $\begin{array}{c}\text { Lenander } \\
\text { et al., 2018, } \\
\text { Sweden [51] }\end{array}$ & $\begin{array}{c}\text { Cross- } \\
\text { sectional }\end{array}$ & Primary care & 1720 & 87.5 & 74.5 & 1 year & 8 weeks & $\begin{array}{l}\text { Discontinuation } \\
\text { of DRPs }\end{array}$ & $\begin{array}{c}\text { Pts with anti- } \\
\text { cholinergics = } \\
9.2 \%\end{array}$ & $\begin{array}{c}\text { Pts with } \\
\text { anticholinergics = } \\
4.2 \%\end{array}$ & + & $\begin{array}{l}\text { Student's } t \text {-test, } \\
\text { Chi-square }\end{array}$ \\
\hline $\begin{array}{l}\text { Weichert } \\
\text { et al., 2018, } \\
\text { Finland [44] }\end{array}$ & $\begin{array}{l}\text { Observational } \\
\text { study }\end{array}$ & Hospital & 549 & 79.6 & 58.3 & $\begin{array}{l}1 \text { year, } 5 \\
\text { months }\end{array}$ & 30 days & $\begin{array}{l}\text { Reduction in } \\
\text { ACB Score } \\
\text { during the } \\
\text { hospital stay }\end{array}$ & $\begin{array}{c}\text { Patients on } \\
\text { DAPs on } \\
\text { admission }= \\
60.8 \%\end{array}$ & $\begin{array}{c}\text { Patients on DAPs } \\
\text { on discharge = } \\
57.7\end{array}$ & - & $\begin{array}{c}\text { Shapiro-Wilk test, } \\
\text { Wilcoxon } \\
\text { signed-rank test,2 } \\
\text { sample } t \text {-test, } \\
\text { Yates and } \\
\text { Pearson's } \\
\text { chi-square test } \\
\text { multivariate } \\
\text { binary logistic } \\
\text { reggression }\end{array}$ \\
\hline $\begin{array}{l}\text { Lenander } \\
\text { et al., 2017 } \\
\text { Sweden [30] }\end{array}$ & $\begin{array}{l}\text { Interventional } \\
\text { pilot study }\end{array}$ & Primary care & $\begin{array}{c}2400 \text { to } 13,700 \\
\text { patients } \\
\text { (estimated) }\end{array}$ & $\begin{array}{c}65-79 \\
\text { (range) }\end{array}$ & 63 & 9 months & 6 months & $\begin{array}{l}\text { Reduction in } \\
\text { anticholiner- } \\
\text { gic PIMs } \\
\text { (before/after) }\end{array}$ & $\begin{array}{l}\text { Anticholinergic } \\
\text { prescriptions } \\
\text { before } \\
\text { intervention } \\
(4513)\end{array}$ & $\begin{array}{c}\text { Anticholinergic } \\
\text { prescriptions after } \\
\text { intervention } \\
(3824)\end{array}$ & + & Chi-square test \\
\hline
\end{tabular}


Table 2. Cont.

\begin{tabular}{|c|c|c|c|c|c|c|c|c|c|c|c|c|}
\hline \multirow{2}{*}{$\begin{array}{l}\text { Author, Year, } \\
\text { Country }\end{array}$} & \multirow{2}{*}{ Study Design } & \multirow{2}{*}{ Setting } & \multirow{2}{*}{ Sample Size } & \multirow{2}{*}{$\begin{array}{c}\text { Mean Age } \\
\text { (Years) }\end{array}$} & \multirow{2}{*}{$\begin{array}{c}\text { Gender } \\
\text { (Female \%) }\end{array}$} & \multirow{2}{*}{$\begin{array}{c}\text { Study } \\
\text { Duration }\end{array}$} & \multirow{2}{*}{ Follow-Up } & \multirow{2}{*}{$\begin{array}{c}\text { Relevant } \\
\text { Outcome(s) }\end{array}$} & \multicolumn{2}{|c|}{ Outcome Measure } & \multirow{2}{*}{$\begin{array}{c}\text { Significant } \\
\text { Association } \\
( \pm)\end{array}$} & \multirow{2}{*}{ Statistical Tests } \\
\hline & & & & & & & & & Control/Pre & Intervention/Post & & \\
\hline $\begin{array}{l}\text { Moga et al., } \\
\text { 2017, USA } \\
\text { [29] }\end{array}$ & $\begin{array}{l}\text { Parallel arm } \\
\text { Randomised } \\
\text { Interventional } \\
\text { study }\end{array}$ & $\begin{array}{l}\text { Alzheimer's } \\
\text { Disease } \\
\text { Center }\end{array}$ & 49 & $77.7 \pm 6.6$ & 70.0 & 1 year & 8 weeks & $\begin{array}{l}\text { Significant } \\
\text { reduction in } \\
\text { anticholiner- } \\
\text { gic drug scale } \\
\text { (ADS) Score }\end{array}$ & $1.0(0.3)$ & $0.2(0.3)$ & + & $\begin{array}{l}\text { Student's } t \text {-tests } \\
\text { (or Wilcoxon } \\
\text { rank-sum tests for } \\
\text { non-normally } \\
\text { distributed } \\
\text { variables), } \\
\text { Chi-square or } \\
\text { Fisher's exact tests }\end{array}$ \\
\hline $\begin{array}{l}\text { Lagrange } \\
\text { et al., 2017, } \\
\text { France [52] }\end{array}$ & $\begin{array}{l}\text { Retrospective } \\
\text { study }\end{array}$ & Hospital & 187 & 73.9 & 63.1 & 10.5 months & $\begin{array}{l}33 \text { and } 37 \\
\text { days }\end{array}$ & $\begin{array}{l}\text { Change in } \\
\text { number of } \\
\text { prescriptions }\end{array}$ & $\begin{array}{c}6538 \text { doses } \\
\text { (Anticholiner- } \\
\text { gics) }\end{array}$ & $\begin{array}{c}4696 \text { doses } \\
\text { (Anticholinergics) }\end{array}$ & + & $\begin{array}{l}\text { Descriptive } \\
\text { statistics }\end{array}$ \\
\hline \multirow{2}{*}{$\begin{array}{l}\text { Carnahan } \\
\text { et al., 2017, } \\
\text { USA [39] }\end{array}$} & \multirow{2}{*}{$\begin{array}{c}\text { Quasi- } \\
\text { experimental } \\
\text { study design }\end{array}$} & \multirow{2}{*}{$\begin{array}{l}\text { Nursing } \\
\text { home }\end{array}$} & \multirow[t]{2}{*}{411} & \multirow[t]{2}{*}{86.7} & \multirow[t]{2}{*}{77.0} & \multirow{2}{*}{$\begin{array}{l}1 \text { year } 9 \\
\text { months }\end{array}$} & \multirow[t]{2}{*}{276 days } & $\begin{array}{l}\text { Anticholinergic } \\
\text { use }\end{array}$ & $\begin{array}{c}\text { Mean (SD) } \\
35.9 \%(12.0 \%) \\
\end{array}$ & $\begin{array}{c}\text { Mean (SD) } \\
36.1 \%(10.9 \%)\end{array}$ & - & \multirow{2}{*}{$\begin{array}{l}\text { Generalised linear } \\
\text { mixed logistic } \\
\text { regression }\end{array}$} \\
\hline & & & & & & & & $\begin{array}{c}\text { Antipsychotic } \\
\text { use }\end{array}$ & $\begin{array}{c}\text { Mean (SD) } \\
17.7 \%(10.4 \%)\end{array}$ & $\begin{array}{c}\text { Mean (SD) } \\
20.7 \%(10.6 \%)\end{array}$ & + & \\
\hline $\begin{array}{c}\text { Hanus et al., } \\
\text { 2016, USA } \\
\text { [40] }\end{array}$ & $\begin{array}{l}\text { Observational } \\
\text { Pilot study }\end{array}$ & $\begin{array}{l}\text { Medical } \\
\text { clinics }\end{array}$ & 59 & $77 \pm 9.3$ & 51.0 & 2 months & 2 weeks & $\begin{array}{l}\text { Reduction in } \\
\text { ACB Score, } \\
\text { Increased } \\
\text { medication } \\
\text { acceptance } \\
\text { rate }\end{array}$ & $\begin{array}{l}1.08 \\
50 \%\end{array}$ & $\begin{array}{l}0.89 \\
95 \%\end{array}$ & + & $\begin{array}{c}\text { Generalised linear } \\
\text { mixed-effects } \\
\text { model, paired } \\
t \text {-test }\end{array}$ \\
\hline $\begin{array}{l}\text { McLarin et al., } \\
\text { 2016, } \\
\text { Australia [43] }\end{array}$ & $\begin{array}{l}\text { Retrospective } \\
\text { study. }\end{array}$ & $\begin{array}{l}\text { Aged care } \\
\text { facilities }\end{array}$ & 814 & 85.6 & 69.6 & NA & NA & $\begin{array}{c}\text { Reduction in } \\
\text { anticholiner- } \\
\text { gic } \\
\text { medications } \\
\text { after a } \\
\text { medication } \\
\text { review }\end{array}$ & $\begin{array}{l}\text { Mean (SD) } \\
3.73(1.46)\end{array}$ & $\begin{array}{l}\text { Mean (SD) } \\
3.32(1.7)\end{array}$ & + & $\begin{array}{l}\text { Wilcoxon } \\
\text { signed-rank test, } \\
\text { ANOVA }\end{array}$ \\
\hline $\begin{array}{l}\text { Kersten et al., } \\
\text { 2015, Norway } \\
\text { [46] }\end{array}$ & $\begin{array}{l}\text { Retrospective } \\
\text { study }\end{array}$ & Hospital & 232 & 86.1 & 59.1 & 8 months & 1 year & $\begin{array}{c}\text { Reduction in } \\
\text { anticholiner- } \\
\text { gic } \\
\text { prescriptions }\end{array}$ & $\begin{array}{l}\text { Prevalence of } \\
\text { was significan }\end{array}$ & $\begin{array}{l}\text { ticholinergic drugs } \\
\text { reduced }(p<0.02)\end{array}$ & + & $\begin{array}{c}\text { Paired samples } \\
\text { Student's } t \text {-test, } \\
\text { McNamar's test, } \\
\text { Mann-Whitney U } \\
\text { tests, ANOVA, } \\
\text { linear regression }\end{array}$ \\
\hline $\begin{array}{l}\text { Juola et al., } \\
\text { 2015, Finland } \\
\text { [45] }\end{array}$ & Cluster RCT & $\begin{array}{l}\text { Assisted } \\
\text { living } \\
\text { facilities }\end{array}$ & 227 & 83.0 & 70.9 & 1 year & 1 year & $\begin{array}{l}\text { Mean Anti- } \\
\text { cholinergic } \\
\text { drugs }\end{array}$ & $\begin{array}{l}1.0 \\
\text { (Mean Anti- } \\
\text { cholinergic } \\
\text { drugs) }\end{array}$ & $\begin{array}{c}1.2 \\
\text { (Mean } \\
\text { Anticholinergic } \\
\text { drugs) }\end{array}$ & - & $\begin{array}{c}\text { tests, } \\
\text { Mann-Whitney U } \\
\text { tests, or } \\
\text { Chi-square tests, } \\
\text { GEE models, } \\
\text { Poisson regression } \\
\text { models }\end{array}$ \\
\hline $\begin{array}{l}\text { Kersten et al., } \\
\text { 2013, Norway } \\
\text { [21] }\end{array}$ & RCT & $\begin{array}{l}\text { Nursing } \\
\text { home }\end{array}$ & 87 & 85.0 & 39.0 & 8 weeks & 8 weeks & $\begin{array}{l}\text { Marked } \\
\text { reduction in } \\
\text { ADS score }\end{array}$ & Median $=4$ & Median $=2$ & + & $\begin{array}{c}\text { ANCOVA, } \\
\text { Poisson regression } \\
\text { analysis }\end{array}$ \\
\hline
\end{tabular}


Table 2. Cont.

\begin{tabular}{|c|c|c|c|c|c|c|c|c|c|c|c|c|}
\hline \multirow{2}{*}{$\begin{array}{l}\text { Author, Year, } \\
\text { Country }\end{array}$} & \multirow{2}{*}{ Study Design } & \multirow{2}{*}{ Setting } & \multirow{2}{*}{ Sample Size } & \multirow{2}{*}{$\begin{array}{c}\text { Mean Age } \\
\text { (Years) }\end{array}$} & \multirow{2}{*}{$\begin{array}{c}\text { Gender } \\
\text { (Female \%) }\end{array}$} & \multirow{2}{*}{$\begin{array}{c}\text { Study } \\
\text { Duration }\end{array}$} & \multirow{2}{*}{ Follow-Up } & \multirow{2}{*}{$\begin{array}{c}\text { Relevant } \\
\text { Outcome(s) }\end{array}$} & \multicolumn{2}{|c|}{ Outcome Measure } & \multirow{2}{*}{$\begin{array}{c}\text { Significant } \\
\text { Association } \\
( \pm)\end{array}$} & \multirow{2}{*}{ Statistical Tests } \\
\hline & & & & & & & & & Control/Pre & Intervention/Post & & \\
\hline $\begin{array}{c}\text { Ghibelli et al., } \\
\text { 2013, Italy } \\
\text { [53] }\end{array}$ & $\begin{array}{l}\text { Pre- and post- } \\
\text { intervention } \\
\text { study }\end{array}$ & Hospital & $\begin{array}{l}75 \text { for Pre } \\
75 \text { for Post }\end{array}$ & 81 & 58.3 & 4 months & NA & $\begin{array}{l}\text { Reduction in } \\
\text { ACB score }\end{array}$ & 1.3 & 1.1 & - & $\begin{array}{c}\text { Pearson } \\
\text { Chi-square test, } \\
\text { Student's } t \text {-test }\end{array}$ \\
\hline $\begin{array}{c}\text { Yeh et al., } \\
\text { 2013, Taiwan } \\
\text { [54] }\end{array}$ & $\begin{array}{l}\text { Prospective } \\
\text { case-control }\end{array}$ & $\begin{array}{l}\text { Veteran } \\
\text { Home }\end{array}$ & 67 & 83.4 & NA & 12 weeks & 12 weeks & $\begin{array}{l}\text { Anticholinergic } \\
\text { Burden } \\
\text { (CR-ACHS) }\end{array}$ & $\begin{array}{c}1.0 \pm 1.1 \\
\text { (Mean } \\
\text { CR-ACHS) }\end{array}$ & $\begin{array}{c}-0.5 \pm 1.1 \\
\text { (Mean CR-ACHS) }\end{array}$ & + & $\begin{array}{l}\text { Wilcoxon signed } \\
\text { ranks test }\end{array}$ \\
\hline $\begin{array}{l}\text { Boustani } \\
\text { et al.,2012, } \\
\text { USA [41] }\end{array}$ & $\mathrm{RCT}$ & Hospital & 424 & 74.8 & 68.0 & 21 months & $\begin{array}{l}\text { At the time } \\
\text { of } \\
\text { discharge }\end{array}$ & $\begin{array}{l}\text { Discontinuation } \\
\text { of AC } \\
\text { prescriptions }\end{array}$ & $\begin{array}{l}\text { anticholinergic } \\
\text { discontinued } \\
\quad=31.2 \%\end{array}$ & $\begin{array}{c}\text { anticholinergic } \\
\text { discontinued = } \\
48.9 \%\end{array}$ & - & $\begin{array}{c}\text { Fisher's exact test, } \\
\text { t-test, logistic } \\
\text { regression, } \\
\text { multiple } \\
\text { regression }\end{array}$ \\
\hline $\begin{array}{l}\text { Gnjidic et al., } \\
2010, \\
\text { Australia [23] }\end{array}$ & Cluster RCT & $\begin{array}{l}\text { Self-care } \\
\text { retirement } \\
\text { village }\end{array}$ & 115 & 84.3 & 73.0 & 13 months & 3 months & $\begin{array}{l}\text { Drug Burden } \\
\text { Index (DBI) }\end{array}$ & $\begin{array}{l}0.26 \pm 0.34 \\
\text { (mean DBI) }\end{array}$ & $0.22 \pm \underset{D B I}{0.42}$ (mean & - & $\begin{array}{c}\text { Kolmogorov- } \\
\text { Smirnov test } \\
\text { Mann-Whitney } \\
\text { nonparametric } \\
\text { test } \\
X^{2} \text { test }\end{array}$ \\
\hline $\begin{array}{c}\text { Castelino } \\
\text { et al., 2010, } \\
\text { Australia [22] }\end{array}$ & $\begin{array}{c}\text { A } \\
\text { retrospective } \\
\text { analysis of } \\
\text { medication } \\
\text { reviews }\end{array}$ & $\begin{array}{l}\text { Community- } \\
\text { dwelling }\end{array}$ & 372 & 76.1 & 55.0 & NA & NA & $\begin{array}{l}\text { Impact of } \\
\text { pharmacist's } \\
\text { on DBI scores }\end{array}$ & $\begin{array}{l}\text { Sum of DBI } \\
\text { scores = } \\
206.86\end{array}$ & $\begin{array}{l}\text { Sum of DBI scores } \\
\quad=157.26\end{array}$ & + & $\begin{array}{l}\text { Wilcoxon } \\
\text { signed-rank test }\end{array}$ \\
\hline $\begin{array}{l}\text { Nishtala et al., } \\
2009, \\
\text { Australia [42] }\end{array}$ & $\begin{array}{l}\text { Retrospective } \\
\text { study }\end{array}$ & $\begin{array}{l}\text { Aged care } \\
\text { homes }\end{array}$ & 500 & 84.0 & 75.0 & 6 months & 2 months & $\begin{array}{l}\text { Significant } \\
\text { decrease in } \\
\text { DBI score }\end{array}$ & NA & $\begin{array}{l}12 \% \text { decrease in } \\
\text { DBI }\end{array}$ & + & $\begin{array}{l}\text { 2-tailed Wilcoxon } \\
\text { signed-rank test }\end{array}$ \\
\hline $\begin{array}{c}\text { van Eijk et al., } \\
\text { 2001, } \\
\text { Netherlands } \\
\text { [55] }\end{array}$ & RCT & Primary care & 46,078 & 71 & 58.0 & 1 year & NA & $\begin{array}{l}\text { Reduction in } \\
\text { the } \\
\text { prescribing of } \\
\text { anticholiner- } \\
\text { gics }\end{array}$ & $\begin{array}{c}30 \% \\
\text { reduction in } \\
\text { the rate of } \\
\text { starting } \\
\text { highly anti- } \\
\text { cholinergic } \\
\text { antidepres- } \\
\text { sant in the } \\
\text { individual } \\
\text { intervention } \\
\text { arms } \\
\text { compared } \\
\text { with the } \\
\text { control arm }\end{array}$ & $\begin{array}{l}40 \% \text { reduction in } \\
\text { the rate of starting } \\
\text { highly } \\
\text { anticholinergic } \\
\text { antidepressants in } \\
\text { the group } \\
\text { intervention arms } \\
\text { compared with } \\
\text { the control arm }\end{array}$ & + & $\begin{array}{l}\text { Poisson regression } \\
\text { model }\end{array}$ \\
\hline
\end{tabular}




\subsection{Methodological Quality of Studies}

All eligible studies were rated for their methodological quality, and many studies ( $n=14,61 \%$ ) were identified to be of good quality based on the Newcastle-Ottawa scale $[22,30,38,39,42-44,46-48,50-53]$ (Table S2). The quality of the RCTs was critically appraised using the Cochrane risk of bias assessment tool as shown in Supplementary Table S3. There was a general lack of adequate blinding between study subjects and healthcare practitioners, and between outcomes and assessors. Nonetheless, the follow-up duration was either not clearly specified or insufficient (less than six months) in many studies [21-23,29,40-44,48,50-55]. Altogether, the studies had a duration of follow-up ranging from 14 days $[40,48]$ to 1 year $[45,46,49]$ (Table 2$)$.

\subsection{Intervention Characteristics}

All studies tested single-component interventions, and medication review was the most common single-component healthcare practitioner-oriented intervention [21-23,42-44,46,48,50,51,54] followed by the provision of education to the healthcare practitioners $[38,39,45,47,54,55]$. Healthcare practitioners conducted medication reviews using patient notes or tools such as drug burden index (DBI) and anticholinergic burden (ACB) [23,42-44,48,50]. Pharmacists implemented interventions without collaboration with other healthcare practitioners in nearly half of the studies $(n=11)$.

Healthcare practitioner-initiated education mainly consisted of professional components, such as academic detailing sessions for physicians [47,54,55], evidence-based training programs to improve dispensing [39], interactive training sessions for nurses [45], and mailing of intervention letters to the physicians [38]. In three studies [21,29,30], healthcare practitioners also performed interventions such as targeted patient-centred, pharmacistphysician team medication therapy management (MTM) intervention, SÄKLÄK project, and multidisciplinary medication review in collaborations with other healthcare practitioners. A context-aware pharmaceutical analysis tool was tested in France to automatically compare prescriptions recorded in computerised patient files against the main consensual guidelines [52]. Another study tested the clinical decision support system to discontinue orders of definite anticholinergic medications for hospitalised patients with cognitive impairment [41]. Similarly, a study tested targeted patient-centred pharmacist-physician team MTM intervention to reduce the consumption of inappropriate anticholinergic medications in older patients [29]. In Italy, researchers tested the INTERcheck computerised prescription support system to optimise drug prescriptions and minimise the occurrence of adverse drug reactions [53].

\subsection{Effectiveness of Interventions at Improving Anticholinergic Prescribing Practice}

Sixteen studies (70\%) $[21,22,29,30,38-40,42,43,46,48,50-52,54,55]$ investigating a healthcare practitioner-oriented intervention reported a significant reduction in anticholinergic prescribing errors, whereas seven studies $(30 \%)[23,41,44,45,47,49,53]$ reported no significant effect (Table 2). Similarly, medication review $(n=8)$ and the provision of education $(n=4)$ were the most common interventions in these sixteen studies; however, these studies varied in their designs. There were 14 studies (87.5\%) $[22,30,38,39,42-44,46-48,50-53]$ that were of high quality, and of those, 11 studies [22,30,38,39,42,43,46,48,50-52] showed a significant reduction in anticholinergic prescribing errors. Seven studies had a follow-up period of $\geq 6$ months, and four studies showed a significant reduction in anticholinergic prescribing errors. With a shorter follow-up period of 2 weeks to 6 months, 4 studies $[42,48,51,52]$ out of 10 studies reported reductions in anticholinergic prescribing errors (Table 2).

Healthcare practitioner-oriented interventions that reported a significant reduction in anticholinergic prescribing errors included: medication review, education provision to healthcare practitioners, pharmacist-led electronic health record-based population health initiative and anticholinergic risk scale service, targeted patient-centred, pharmacistphysician team MTM intervention, context-aware pharmaceutical analysis tool, and SÄKLÄK project. Healthcare practitioner-oriented interventions were most effective in reducing ACB $[21,29,40,54]$, DBI $[22,42,48,50]$, and discontinuation or reduction of anticholinergic 
medications [30,38,39,43,46,51,52,55]. Hernandez et al. 2020 reported a decline in DBI from 1.38 (control group) to 1.08 (intervention group) [50]. Another study reported a reduction in ACB score from 1.08 (control group) to 0.89 (intervention group) [40]. A retrospective study by McLarin et al. [43] in Australia found a reduction in the mean scores of anticholinergic medications from 3.73 to 3.02 after implementing medication review.

\section{Discussion}

This is believed to be the first systematic review assessing the effectiveness of interventions to reduce anticholinergic prescribing errors in adults aged 65 and above. Previous reviews primarily evaluated the studies of pharmacist-oriented interventions on medication prescribing and the association between anticholinergic drug burden and mortality in older people $[17,56,57]$. We did not conduct a meta-analysis because of the methodological heterogeneity between the study designs, anticholinergic prescribing errors, types of interventions, study duration, and follow-up period. Given the high prevalence of inappropriate prescribing and polypharmacy in older people aged 65 and over, interventions to reduce anticholinergic prescribing errors in this cohort are of considerable importance. This systematic review identified 23 studies reporting interventions to reduce anticholinergic prescribing errors in older people. The interventions were mainly provided by the pharmacists using a patient-centred approach. Many studies (19 out of 23) successfully reduced the incidence of anticholinergic prescribing errors in older people. Evidence related to the pharmacist-led interventions in many studies suggests that pharmacists play a vital role in the care of older people, thus improving medication safety across the continuum of care.

In this study, medication review and education provision to the healthcare practitioners were the most common elements in many interventions. Medication review is a structured evaluation of patients' pharmacotherapy to optimise drug use and reduce the occurrence of drug-related problems [58]. Similarly, medication review is recognised as an important healthcare practitioner-oriented intervention for reducing anticholinergic prescribing errors in older people [59]. Likewise, older people benefit mostly from medication reviews as this cohort is more susceptible to adverse drug effects $[60,61]$. The efficacy of medication review in reducing anticholinergic prescribing errors was reported by eight studies in this review $[15,21,22,43,44,46,50,62]$. Previous studies inform the significant effects of structured medication review on medication prescriptions and older adults' quality of life [63-65].

Another intervention, such as the provision of education to the healthcare practitioners, was tested in eight studies, but only five studies reported the effectiveness of this intervention in reducing anticholinergic prescribing errors in older people $[38,49,54,55,66]$. Evidence informs that the healthcare practitioner-oriented educational intervention effectively reduces prescribing errors in older people $[57,67]$. The provision of education reduces the use of healthcare resources, including emergency department presentations and hospital admissions [68]. Implementing healthcare practitioner-led educational interventions encourages prescribers to change prescription practices, thus improving prescribers' clinical practice [69]. An education intervention provides precise knowledge about prescribing in older adults, medication-related errors, and prevention strategies for reducing medication-related errors [69]. This review also showed that interventions such as INTERcheck, SÄKLÄK intervention model, targeted MTM intervention, context-aware pharmaceutical analysis tool, and CDSS alert were not successful in reducing anticholinergic prescribing errors in older adults $[29,41,51-53]$.

\subsection{Implications for Clinical Practice and Future Research}

Medications with anticholinergic activity are frequently prescribed in older people due to their numerous clinical benefits; however, these medications are also associated with poor clinical outcomes [70]. Implementing healthcare practitioner-oriented interventions can reduce the occurrence of anticholinergic prescribing errors in older people. This review's findings inform that healthcare practitioner-oriented interventions appear to improve medication safety in older people based on observed reductions in anticholinergic 
prescribing errors, particularly when the provision of care involves a medication review and an education for the physicians prescribing anticholinergic medications. The prescribing competency can be optimised through educational interventions [71], thus reducing the occurrence of anticholinergic prescribing errors. The safe and effective prescribing of medications is a challenge in older people who frequently experience multiple long-term conditions and complex polypharmacy [72]. Due to an increasing challenge to physicians when prescribing and the complexity of medication regimens taken by older people, there is a need to embed prescribing competency framework in clinical practice [72]. The prescribing competency framework engages prescribers in different stages of prescribing, such as information gathering, clinical decision making, communication, monitoring, and review [72].

The current quantification methods for anticholinergic burden tend to streamline the complexity of pharmacological mechanisms in geriatric risk assessment in older adults. However, there is no universally accepted quantification method available to estimate anticholinergic drug burden, and it is difficult to compare the study findings from distinct methods [16]. Existing tools derived from expert consensus limit the quantification of anticholinergic burden as they do not take into consideration the dose and the CNS distribution of drugs [15]. A recent review showed that the ratings of anticholinergic activity in the expert opinion scales were inconsistent [73]. Moreover, the estimation of central cognitive effects by measuring in vitro serum assay of medications with known anticholinergic activity as a composite peripheral measure still remains unclear [16]. The lack of a gold standard method for anticholinergic quantification might have a direct or indirect impact on the interpretation of the effect size of the study interventions (e.g., reduction in anticholinergic burden). In this review, many studies (69\%) were either cross-sectional or nonrandomised and included a single-component intervention. Therefore, there is a need to conduct future randomised multicomponent intervention trials for evaluating the true impact of healthcare practitioner-oriented interventions on anticholinergic prescribing errors in older people.

\subsection{Strengths and Limitations}

This study was comprehensive in that the electronic search, conducted in four important databases, attempted to identify the complete existing body of evidence of the effectiveness of healthcare-oriented interventions aimed at reducing anticholinergic prescribing errors in older people. This is the first review that found 17 different types of healthcare-oriented interventions, and their impact on anticholinergic prescribing errors. A limitation of this study included the absence of meta-analysis and the estimation of the effect size. It was mainly due to the heterogeneity of included studies. We also excluded studies published in languages other than English, which may have introduced a language bias. However, we performed citation tracking and hand-searching of all included studies to minimise the influence of factors (e.g., inconsistent terminology or wrong indexing) that may affect the keyword-based search.

\section{Conclusions}

This systematic review suggests that healthcare practitioner-oriented interventions have the potential to reduce the occurrence of anticholinergic prescribing errors in older people. Medication review and the provision of education to the prescribers were the most common approaches to reducing anticholinergic prescribing errors in older people. Healthcare practitioner-oriented interventions were mostly effective in reducing the burden of anticholinergic medications and facilitating the discontinuation of anticholinergic medications in older people. In the future, there is also the need to ascertain how often the healthcare practitioner performs interventions that may reduce anticholinergic prescribing errors in older people. 
Supplementary Materials: The following supporting information can be downloaded at: https: / / www.mdpi.com/article/10.3390/jcm11030714/s1, Table S1: MEDLINE search strategy; Table S2: The Newcastle-Ottawa scale risk of bias assessment for included cohort studies; Table S3: The Cochrane Risk of Bias tool results for included RCTs.

Author Contributions: Conceptualisation, M.S.S. and S.A. (Sheraz Ali); methodology, M.S.S. and S.A. (Sheraz Ali); validation, M.S.S. and S.A. (Sheraz Ali); formal analysis, S.A. (Sana Ajaz), M.A., A.A., S.A. (Saud Alotaibi), M.F. and A.F.; data curation, S.A. (Sana Ajaz), M.A., A.A., S.A. (Saud Alotaibi), M.F. and A.F.; writing - original draft preparation, S.A. (Sheraz Ali); writing-review and editing, S.A. (Sheraz Ali) and M.S.S.; supervision, M.S.S.; project administration, M.S.S. and S.A. (Sheraz Ali). All authors have read and agreed to the published version of the manuscript.

Funding: This research received no specific grant from any funding agency in public, commercial, or not-for-profit sectors.

Institutional Review Board Statement: Not applicable.

Informed Consent Statement: Not applicable.

Data Availability Statement: All research data generated or analysed during this review are included in this published article.

Conflicts of Interest: The authors declare no conflict of interest.

\section{References}

1. Buxton, I.L.O. Principles of Prescription Order Writing and Patient Compliance. In Goodman Eamp, Gilman's: The Pharmacological Basis of Therapeutics, 12th ed.; Brunton, L.L., Chabner, B.A., Knollmann, B.C., Eds.; McGraw-Hill Education: New York, NY, USA, 2015.

2. Likic, R.; Maxwell, S.R. Prevention of medication errors: Teaching and training. Br. J. Clin. Pharmacol. 2009, 67, 656-661. [CrossRef] [PubMed]

3. Drag, L.L.; Wright, S.L.; Bieliauskas, L.A. Prescribing Practices of Anticholinergic Medications and Their Association with Cognition in an Extended Care Setting. J. Appl. Gerontol. 2012, 31, 239-259. [CrossRef]

4. Barton, C.; Sklenicka, J.; Sayegh, P.; Yaffe, K. Contraindicated medication use among patients in a memory disorders clinic. Am. J. Geriatr. Pharmacother. 2008, 6, 147-152. [CrossRef] [PubMed]

5. Buck, M.D.; Atreja, A.; Brunker, C.P.; Jain, A.; Suh, T.T.; Palmer, R.M.; Dorr, D.A.; Harris, C.M.; Wilcox, A.B. Potentially inappropriate medication prescribing in outpatient practices: Prevalence and patient characteristics based on electronic health records. Am. J. Geriatr. Pharmacother. 2009, 7, 84-92. [CrossRef]

6. Corsonello, A.; Pedone, C.; Lattanzio, F.; Lucchetti, M.; Garasto, S.; Di Muzio, M.; Giunta, S.; Onder, G.; Di Iorio, A.; Volpato, S.; et al. Potentially Inappropriate Medications and Functional Decline in Elderly Hospitalized Patients. J. Am. Geriatr. Soc. 2009, 57, 1007-1014. [CrossRef] [PubMed]

7. Hale, L.; Griffin, A.; Cartwright, O.; Moulin, J.; Alford, S.; Fleming, R. Potentially inappropriate medication use in hospitalized older adults: A DUE using the full Beers criteria. Formulary 2008, 43, 326-336.

8. Rajska-Neumann, A.; Wieczorowska-Tobis, K. Polypharmacy and potential inappropriateness of pharmaco-logical treatment among community-dwelling elderly patients. Arch. Gerontol. Geriatr. 2007, 44 (Suppl. S1), 303-309. [CrossRef]

9. Gallagher, P.F.; Barry, P.J.; Ryan, C.; Hartigan, I.; O’Mahony, D. Inappropriate prescribing in an acutely ill population of elderly patients as determined by Beers' Criteria. Age Ageing 2008, 37, 96-101. [CrossRef]

10. Ruxton, K.; Woodman, R.J.; Mangoni, A.A. Drugs with anticholinergic effects and cognitive impairment, falls and all-cause mortality in older adults: A systematic review and meta-analysis. Br. J. Clin. Pharmacol. 2015, 80, 209-220. [CrossRef]

11. Van Eijk, M.E.; Bahri, P.; Dekker, G.; Herings, R.M.; Porsius, A.; Avorn, J.; De Boer, A. Use of prevalence and incidence measures to describe age-related prescribing of antidepressants with and without anticholinergic effects. J. Clin. Epidemiol. 2000, 53, 645-651. [CrossRef]

12. Campbell, N.; Boustani, M.; Limbil, T.; Ott, C.; Fox, C.; Maidment, I.; Schubert, C.C.; Munger, S.; Fick, D.; Miller, D.; et al. The cognitive impact of anticholinergics: A clinical review. Clin. Interv. Aging 2009, 4, 225-233. [PubMed]

13. Rudolph, J.L.; Salow, M.J.; Angelini, M.C.; McGlinchey, R.E. The anticholinergic risk scale and anticholinergic adverse effects in older persons. Arch. Intern. Med. 2008, 168, 508-513. [CrossRef]

14. Kersten, H.; Wyller, T.B. Anticholinergic drug burden in older people's brain-How well is it measured? Basic Clin. Pharmacol. Toxicol. 2014, 114, 151-159. [CrossRef] [PubMed]

15. Nishtala, P.S.; Salahudeen, M.S.; Hilmer, S.N. Anticholinergics: Theoretical and clinical overview. Expert Opin. Drug. Saf. 2016, 15, 753-768. [CrossRef] [PubMed]

16. Salahudeen, M.S.; Nishtala, P.S. Examination and Estimation of Anticholinergic Burden: Current Trends and Implications for Future Research. Drugs Aging 2016, 33, 305-313. [CrossRef] [PubMed] 
17. Ali, S.; Peterson, G.M.; Bereznicki, L.R.; Salahudeen, M.S. Association between anticholinergic drug burden and mortality in older people: A systematic review. Eur. J. Clin. Pharmacol. 2020, 76, 319-335. [CrossRef] [PubMed]

18. Tune, L.E. Anticholinergic effects of medication in elderly patients. J. Clin. Psychiatry 2001, 62 (Suppl. S21), 11-14.

19. Alharafsheh, A.; Alsheikh, M.; Ali, S.; Baraiki, A.A.; Alharbi, G.; Alhabshi, T.; Aboutaleb, A. A retrospective cross-sectional study of antibiotics prescribing patterns in admitted patients at a tertiary care setting in the KSA. Int. J. Health Sci. 2018, 12, 67-71.

20. Gorup, E.; Rifel, J.; Petek Šter, M. Anticholinergic Burden and Most Common Anticholinergic-acting Medicines in Older General Practice Patients. Zdr Varst. 2018, 57, 140-147. [CrossRef]

21. Kersten, H.; Molden, E.; Tolo, I.K.; Skovlund, E.; Engedal, K.; Wyller, T.B. Cognitive effects of reducing anticholinergic drug burden in a frail elderly population: A randomized controlled trial. J. Gerontol. A. Biol. Sci. Med. Sci. 2013, 68, 271-278. [CrossRef]

22. Castelino, R.L.; Hilmer, S.N.; Bajorek, B.V.; Nishtala, P.; Chen, T.F. Drug Burden Index and potentially inappropriate medications in community-dwelling older people: The impact of Home Medicines Review. Drugs Aging 2010, 27, 135-148. [CrossRef] [PubMed]

23. Gnjidic, D.; Le Couteur, D.G.; Abernethy, D.R.; Hilmer, S.N. A pilot randomized clinical trial utilizing the drug burden index to reduce exposure to anticholinergic and sedative medications in older people. Ann. Pharmacother. 2010, 44, 1725-1732. [CrossRef] [PubMed]

24. Tay, H.S.; Soiza, R.L.; Mangoni, A.A. Minimizing anticholinergic drug prescribing in older hospitalized patients: A full audit cycle. Ther. Adv. Drug Saf. 2014, 5, 121-128. [CrossRef] [PubMed]

25. van der Meer, H.G.; Wouters, H.; Pont, L.G.; Taxis, K. Reducing the anticholinergic and sedative load in older patients on polypharmacy by pharmacist-led medication review: A randomised controlled trial. BMJ Open 2018, 8, e019042. [CrossRef]

26. Christensen, M.; Lundh, A. Medication review in hospitalised patients to reduce morbidity and mortality. Cochrane Database Syst. Rev. 2016, 2, Cd008986. [CrossRef]

27. Holland, R.; Desborough, J.; Goodyer, L.; Hall, S.; Wright, D.; Loke, Y.K. Does pharmacist-led medication review help to reduce hospital admissions and deaths in older people? A systematic review and meta-analysis. Br. J. Clin. Pharmacol. 2008, 65, 303-316. [CrossRef]

28. Wallerstedt, S.M.; Kindblom, J.M.; Nylén, K.; Samuelsson, O.; Strandell, A. Medication reviews for nursing home residents to reduce mortality and hospitalization: Systematic review and meta-analysis. Br. J. Clin. Pharmacol. 2014, 78, 488-497. [CrossRef]

29. Moga, D.C.; Abner, E.L.; Rigsby, D.N.; Eckmann, L.; Huffmyer, M.; Murphy, R.R.; Coy, B.B.; Jicha, G.A. Optimizing medication appropriateness in older adults: A randomized clinical interventional trial to decrease anticholinergic burden. Alzheimers Res. Ther. 2017, 9, 36. [CrossRef]

30. Lenander, C.; Bondesson, Å.; Viberg, N.; Jakobsson, U.; Beckman, A.; Midlöv, P. Effects of an intervention (SÄKLÄK) on prescription of potentially inappropriate medication in elderly patients. Fam. Pract. 2017, 34, 213-218. [CrossRef]

31. Aronson, J.K. Medication errors: Definitions and classification. Br. J. Clin. Pharmacol. 2009, 67, 599-604. [CrossRef]

32. Velo, G.P.; Minuz, P. Medication errors: Prescribing faults and prescription errors. Br. J. Clin. Pharmacol. 2009, 67, 624-628. [CrossRef] [PubMed]

33. Moher, D.; Liberati, A.; Tetzlaff, J.; Altman, D.G. Preferred reporting items for systematic reviews and meta-analyses: The PRISMA statement. PLoS Med. 2009, 6, e1000097. [CrossRef] [PubMed]

34. Ali, S.; Salahudeen, M.S.; Bereznicki, L.R.E.; Curtain, C.M. Pharmacist-led interventions to reduce adverse drug events in older people living in residential aged care facilities: A systematic review. Br. J. Clin. Pharmacol. 2021, 87, 3672-3689. [CrossRef] [PubMed]

35. Guise, J.M.; Chang, C.; Viswanathan, M.; Glick, S.; Treadwell, J.; Umscheid, C.A.; Whitlock, E.; Fu, R.; Berliner, E.; Paynter, R.; et al. Agency for Healthcare Research and Quality Evidence-based Practice Center methods for systematically reviewing complex multicomponent health care interventions. J. Clin. Epidemiol. 2014, 67, 1181-1191. [CrossRef]

36. Higgins, J.P.T.; Altman, D.G. Assessing risk of bias in included studies. In Cochrane Handbook for Systematic Reviews of Interventions; Higgins, J.P.T., Green, S., Eds.; Wiley-Blackwell: Chichester, UK, 2011; pp. 187-242.

37. Wells, G.A.; O'Connell, B.S.D.; Peterson, J.; Welch, V.; Losos, M.; Tugwell, P. The Newcastle-Ottawa Scale (NOS) for Assessing the Quality of Nonrandomized Studies in Meta-Analysis. Available online: www.ohri.ca/programs/clinical_epidemiology/oxford. asp (accessed on 25 December 2021).

38. Starner, C.I.; Norman, S.A.; Reynolds, R.G.; Gleason, P.P. Effect of a retrospective drug utilization review on potentially inappropriate prescribing in the elderly. Am. J. Geriatr. Pharmacother. 2009, 7, 11-19. [CrossRef]

39. Carnahan, R.M.; Brown, G.D.; Letuchy, E.M.; Rubenstein, L.M.; Gryzlak, B.M.; Smith, M.; Reist, J.C.; Kelly, M.W.; Schultz, S.K.; Weckmann, M.T.; et al. Impact of programs to reduce antipsychotic and anticholinergic use in nursing homes. Alzheimers Dement. 2017, 3, 553-561. [CrossRef]

40. Hanus, R.J.; Lisowe, K.S.; Eickhoff, J.C.; Kieser, M.A.; Statz-Paynter, J.L.; Zorek, J.A. Evaluation of a pharmacist-led pilot service based on the anticholinergic risk scale. J. Am. Pharm. Assoc. 2016, 56, 555-561. [CrossRef]

41. Boustani, M.A.; Campbell, N.L.; Khan, B.A.; Abernathy, G.; Zawahiri, M.; Campbell, T.; Tricker, J.; Hui, S.L.; Buckley, J.D.; Perkins, A.J.; et al. Enhancing care for hospitalized older adults with cognitive impairment: A randomized controlled trial. J. Gen. Intern. Med. 2012, 27, 561-567. [CrossRef]

42. Nishtala, P.S.; Hilmer, S.N.; McLachlan, A.J.; Hannan, P.J.; Chen, T.F. Impact of residential medication management reviews on drug burden index in aged-care homes: A retrospective analysis. Drugs Aging 2009, 26, 677-686. [CrossRef] 
43. McLarin, P.E.; Peterson, G.M.; Curtain, C.M.; Nishtala, P.S.; Hannan, P.J.; Castelino, R.L. Impact of residential medication management reviews on anticholinergic burden in aged care residents. Curr. Med. Res. Opin. 2016, 32, 123-131. [CrossRef]

44. Weichert, I.; Romero-Ortuno, R.; Tolonen, J.; Soe, T.; Lebus, C.; Choudhury, S.; Nadarajah, C.V.; Nanayakkara, P.; Orrù, M.; Di Somma, S.; et al. Anticholinergic medications in patients admitted with cognitive impairment or falls (AMiCI). The impact of hospital admission on anticholinergic cognitive medication burden. Results of a multicentre observational study. J. Clin. Pharm. Ther. 2018, 43, 682-694. [CrossRef] [PubMed]

45. Juola, A.L.; Bjorkman, M.P.; Pylkkanen, S.; Finne-Soveri, H.; Soini, H.; Kautiainen, H.; Bell, J.S.; Pitkala, K.H. Nurse Education to Reduce Harmful Medication Use in Assisted Living Facilities: Effects of a Randomized Controlled Trial on Falls and Cognition. Drugs Aging 2015, 32, 947-955. [CrossRef] [PubMed]

46. Kersten, H.; Hvidsten, L.T.; Gløersen, G.; Wyller, T.B.; Wang-Hansen, M.S. Clinical impact of potentially inappropriate medications during hospitalization of acutely ill older patients with multimorbidity. Scand. J. Prim. Health Care 2015, 33, 243-251. [CrossRef] [PubMed]

47. Riordan, D.O.; Hurley, E.; Sinnott, C.; Galvin, R.; Dalton, K.; Kearney, P.M.; Halpin, J.D.; Byrne, S. Pharmacist-led academic detailing intervention in primary care: A mixed methods feasibility study. Int. J. Clin. Pharm. 2019, 41, 574-582. [CrossRef]

48. Ailabouni, N.; Mangin, D.; Nishtala, P.S. DEFEAT-polypharmacy: Deprescribing anticholinergic and sedative medicines feasibility trial in residential aged care facilities. Int. J. Clin. Pharm. 2019, 41, 167-178. [CrossRef] [PubMed]

49. Toivo, T.; Airaksinen, M.; Dimitrow, M.; Savela, E.; Pelkonen, K.; Kiuru, V.; Suominen, T.; Uunimäki, M.; Kivelä, S.L.; Leikola, S.; et al. Enhanced coordination of care to reduce medication risks in older home care clients in primary care: A randomized controlled trial. BMC Geriatr. 2019, 19, 332. [CrossRef]

50. Hernandez, M.; Mestres, C.; Junyent, J.; Costa-Tutusaus, L.; Modamio, P.; Lastra, C.F.; Mariño, E.L. Effects of a multifaceted intervention in psychogeriatric patients: One-year prospective study. Eur. J. Hosp. Pharm. 2020, 27, 226-231. [CrossRef]

51. Lenander, C.; Bondesson, Å.; Viberg, N.; Beckman, A.; Midlöv, P. Effects of medication reviews on use of potentially inappropriate medications in elderly patients, a cross-sectional study in Swedish primary care. BMC Health Serv. Res. 2018, 18, 616. [CrossRef]

52. Lagrange, F.; Lagrange, J.; Bennaga, C.; Taloub, F.; Keddi, M.; Dumoulin, B. A context-aware decision-support system in clinical pharmacy: Drug monitoring in the elderly. Le Pharmacien Hospitalier et Clinicien 2017, 52, 100-110. [CrossRef]

53. Ghibelli, S.; Marengoni, A.; Djade, C.D.; Nobili, A.; Tettamanti, M.; Franchi, C.; Caccia, S.; Giovarruscio, F.; Remuzzi, A.; Pasina, L. Prevention of inappropriate prescribing in hospitalized older patients using a computerized prescription support system (INTERcheck $\left({ }^{\circledR}\right)$ ). Drugs Aging 2013, 30, 821-828. [CrossRef]

54. Yeh, Y.C.; Liu, C.L.; Peng, L.N.; Lin, M.H.; Chen, L.K. Potential benefits of reducing medication-related anticholinergic burden for demented older adults: A prospective cohort study. Geriatr. Gerontol. Int. 2013, 13, 694-700. [CrossRef] [PubMed]

55. van Eijk, M.E.; Avorn, J.; Porsius, A.J.; de Boer, A. Reducing prescribing of highly anticholinergic antidepressants for elderly people: Randomised trial of group versus individual academic detailing. BMJ 2001, 322, 654-657. [CrossRef] [PubMed]

56. Fox, C.; Smith, T.; Maidment, I.; Chan, W.Y.; Bua, N.; Myint, P.K.; Boustani, M.; Kwok, C.S.; Glover, M.; Koopmans, I.; et al. Effect of medications with anti-cholinergic properties on cognitive function, delirium, physical function and mortality: A systematic review. Age Ageing 2014, 43, 604-615. [CrossRef] [PubMed]

57. Riordan, D.O.; Walsh, K.A.; Galvin, R.; Sinnott, C.; Kearney, P.M.; Byrne, S. The effect of pharmacist-led interventions in optimising prescribing in older adults in primary care: A systematic review. SAGE Open Med. 2016, 4, 2050312116652568. [CrossRef]

58. Øyane, N.M.F.; Finckenhagen, M.; Ruths, S.; Thue, G.; Lindahl, A.K. Improving drug prescription in general practice using a novel quality improvement model. Scand. J. Prim. Health Care. 2021, 39, 174-183. [CrossRef]

59. van der Meer, H.G.; Wouters, H.; van Hulten, R.; Pras, N.; Taxis, K. Decreasing the load? Is a Multidisciplinary Multistep Medication Review in older people an effective intervention to reduce a patient's Drug Burden Index? Protocol of a randomised controlled trial. BMJ Open 2015, 5, e009213. [CrossRef]

60. Gurwitz, J.H.; Field, T.S.; Judge, J.; Rochon, P.; Harrold, L.R.; Cadoret, C.; Lee, M.; White, K.; LaPrino, J.; Erramuspe-Mainard, J.; et al. The incidence of adverse drug events in two large academic long-term care facilities. Am. J. Med. 2005, 118, 251-258. [CrossRef]

61. Martin, R.M.; Lunec, S.G.; Rink, E. UK postal survey of pharmacists working with general practices on prescribing issues: Characteristics, roles and working arrangements. Int. J. Pharm. Pract. 1998, 6, 133-139. [CrossRef]

62. Veggeland, T.; Dyb, S. The contribution of a clinical pharmacist to the improvement of medication at a geriatric hospital unit in Norway. Pharm. Pract. 2008, 6, 20-24. [CrossRef]

63. Romskaug, R.; Skovlund, E.; Straand, J.; Molden, E.; Kersten, H.; Pitkala, K.H.; Lundqvist, C.; Wyller, T.B. Effect of Clinical Geriatric Assessments and Collaborative Medication Reviews by Geriatrician and Family Physician for Improving Health-Related Quality of Life in Home-Dwelling Older Patients Receiving Polypharmacy: A Cluster Randomized Clinical Trial. JAMA Intern. Med. 2020, 180, 181-189. [CrossRef]

64. Gallagher, P.F.; O'Connor, M.N.; O'Mahony, D. Prevention of potentially inappropriate prescribing for elderly patients: A randomized controlled trial using STOPP/START criteria. Clin. Pharmacol. Ther. 2011, 89, 845-854. [CrossRef] [PubMed]

65. Clyne, B.; Smith, S.M.; Hughes, C.M.; Boland, F.; Bradley, M.C.; Cooper, J.A.; Fahey, T. Effectiveness of a Multifaceted Intervention for Potentially Inappropriate Prescribing in Older Patients in Primary Care: A Cluster-Randomized Controlled Trial (OPTI-SCRIPT Study). Ann. Fam. Med. 2015, 13, 545-553. [CrossRef] [PubMed] 
66. Pasina, L.; Marengoni, A.; Ghibelli, S.; Suardi, F.; Djade, C.D.; Nobili, A.; Franchi, C.; Guerrini, G. A Multicomponent Intervention to Optimize Psychotropic Drug Prescription in Elderly Nursing Home Residents: An Italian Multicenter, Prospective, Pilot Study. Drugs Aging 2016, 33, 143-149. [CrossRef] [PubMed]

67. Jaam, M.; Naseralallah, L.M.; Hussain, T.A.; Pawluk, S.A. Pharmacist-led educational interventions provided to healthcare providers to reduce medication errors: A systematic review and meta-analysis. PLoS ONE 2021, 16, e0253588. [CrossRef]

68. García-Gollarte, F.; Baleriola-Júlvez, J.; Ferrero-López, I.; Cuenllas-Díaz, Á.; Cruz-Jentoft, A.J. An educational intervention on drug use in nursing homes improves health outcomes resource utilization and reduces inappropriate drug prescription. J. Am. Med. Dir. Assoc. 2014, 15, 885-891. [CrossRef]

69. Trivalle, C.; Cartier, T.; Verny, C.; Mathieu, A.M.; Davrinche, P.; Agostini, H.; Becquemont, L.; Demolis, P. Identifying and preventing adverse drug events in elderly hospitalised patients: A randomised trial of a program to reduce adverse drug effects. J. Nutr. Health Aging 2010, 14, 57-61. [CrossRef]

70. López-Álvarez, J.; Sevilla-Llewellyn-Jones, J.; Agüera-Ortiz, L. Anticholinergic Drugs in Geriatric Psychopharmacology. Front Neurosci. 2019, 13, 309. [CrossRef]

71. Kamarudin, G.; Penm, J.; Chaar, B.; Moles, R. Educational interventions to improve prescribing competency: A systematic review. BMJ Open 2013, 3, e003291. [CrossRef]

72. Picton, C.; Loughrey, C.; Webb, A. The need for a prescribing competency framework to address the burden of complex polypharmacy among multiple long-term conditions. Clin. Med. 2016, 16, 470-474. [CrossRef]

73. Salahudeen, M.S.; Duffull, S.B.; Nishtala, P.S. Anticholinergic burden quantified by anticholinergic risk scales and adverse outcomes in older people: A systematic review. BMC Geriatr. 2015, 15, 31. [CrossRef] 